\title{
Comprehensive molecular profiling of lung adenocarcinoma
}

The Cancer Genome Atlas Research Network*

Adenocarcinoma of the lung is the leading cause of cancer death worldwide. Here we report molecular profiling of 230 resected lung adenocarcinomas using messenger RNA, microRNA and DNA sequencing integrated with copy number, methylation and proteomic analyses. High rates of somatic mutation were seen (mean 8.9 mutations per megabase). Eighteen genes were statistically significantly mutated, including RIT1 activating mutations and newly described loss-of-function MGA mutations which are mutually exclusive with focal MYC amplification. EGFR mutations were more frequent in female patients, whereas mutations in RBM10 were more common in males. Aberrations in NF1, MET, ERBB2 and RIT1 occurred in $13 \%$ of cases and were enriched in samples otherwise lacking an activated oncogene, suggesting a driver role for these events in certain tumours. DNA and mRNA sequence from the same tumour highlighted splicing alterations driven by somatic genomic changes, including exon 14 skipping in MET mRNA in $4 \%$ of cases. MAPK and PI(3)K pathway activity, when measured at the protein level, was explained by known mutations in only a fraction of cases, suggesting additional, unexplained mechanisms of pathway activation. These data establish a foundation for classification and further investigations of lung adenocarcinoma molecular pathogenesis.

Lung cancer is the most common cause of global cancer-related mortality, leading to over a million deaths each year and adenocarcinoma is its most common histological type. Smoking is the major cause of lung adenocarcinoma but, as smoking rates decrease, proportionally more cases occur in never-smokers (defined as less than 100 cigarettes in a lifetime). Recently, molecularly targeted therapies have dramatically improved treatment for patients whose tumours harbour somatically activated oncogenes such as mutant $E G F R^{1}$ or translocated $A L K, R E T$, or ROS1 (refs 2-4). Mutant $B R A F$ and $E R B B 2$ (ref. 5) are also investigational targets. However, most lung adenocarcinomas either lack an identifiable driver oncogene, or harbour mutations in KRAS and are therefore still treated with conventional chemotherapy. Tumour suppressor gene abnormalities, such as those in TP53 (ref. 6), STK11 (ref. 7), CDKN2A ${ }^{8}, K E A P 1$ (ref. 9), and $S M A R C A 4$ (ref. 10) are also common but are not currently clinically actionable. Finally, lung adenocarcinoma shows high rates of somatic mutation and genomic rearrangement, challenging identification of all but the most frequent driver gene alterations because of a large burden of passenger events per tumour genome ${ }^{11-13}$. Our efforts focused on comprehensive, multiplatform analysis of lung adenocarcinoma, with attention towards pathobiology and clinically actionable events.

\section{Clinical samples and histopathologic data}

We analysed tumour and matched normal material from 230 previously untreated lung adenocarcinoma patients who provided informed consent (Supplementary Table 1). All major histologic types of lung adenocarcinoma were represented: $5 \%$ lepidic, 33\% acinar, $9 \%$ papillary, $14 \%$ micropapillary, $25 \%$ solid, $4 \%$ invasive mucinous, $0.4 \%$ colloid and $8 \%$ unclassifiable adenocarcinoma (Supplementary Fig. 1) ${ }^{14}$. Median follow-up was 19 months, and 163 patients were alive at the time of last follow-up. Eighty-one percent of patients reported past or present smoking. Supplementary Table 2 summarizes demographics. DNA, RNA and protein were extracted from specimens and quality-control assessments were performed as described previously ${ }^{15}$. Supplementary Table 3 summarizes molecular estimates of tumour cellularity ${ }^{16}$.
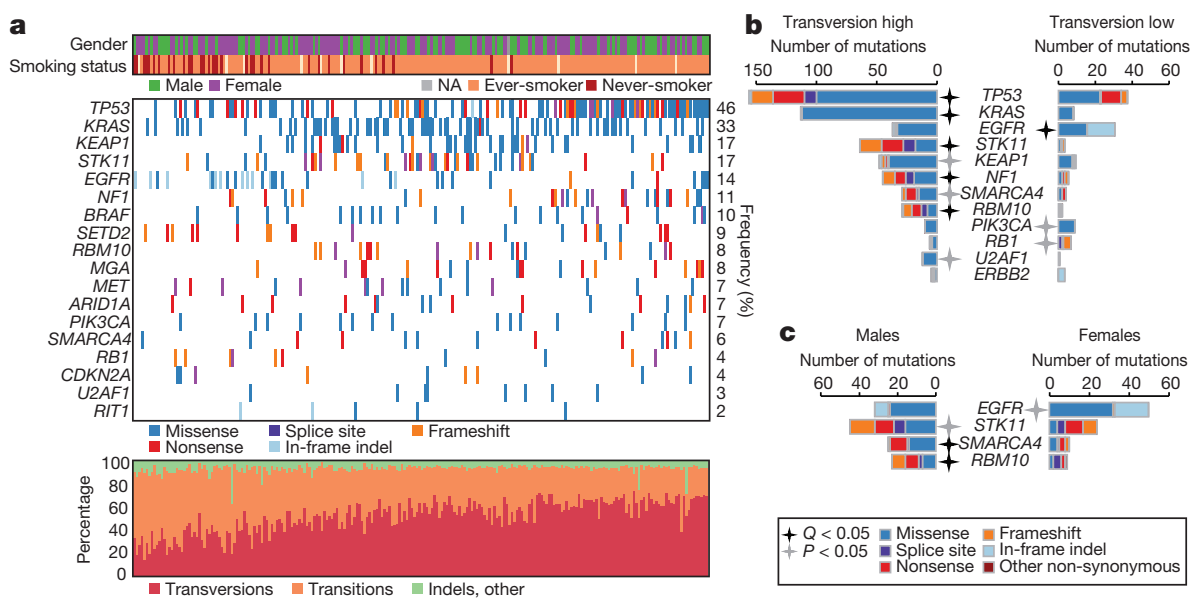

Figure $1 \mid$ Somatic mutations in lung adenocarcinoma. a, Co-mutation plot from whole exome sequencing of 230 lung adenocarcinomas. Data from TCGA samples were combined with previously published data ${ }^{12}$ for statistical analysis. Co-mutation plot for all samples used in the statistical analysis $(n=412)$ can be found in Supplementary Fig. 2. Significant genes with a corrected $P$ value less than 0.025 were identified using the MutSig2CV algorithm and are ranked in order of decreasing prevalence. $\mathbf{b}, \mathbf{c}$, The differential patterns of mutation between samples classified as transversion high and transversion low samples (b) or male and female patients (c) are shown for all samples used in the statistical analysis $(n=412)$. Stars indicate statistical significance using the Fisher's exact test (black stars: $q<0.05$, grey stars: $P<0.05$ ) and are adjacent to the sample set with the higher percentage of mutated samples.

*A list of authors and affiliations appears at the end of the paper. 


\section{Somatically acquired DNA alterations}

We performed whole-exome sequencing (WES) on tumour and germline DNA, with a mean coverage of $97.6 \times$ and $95.8 \times$, respectively, as performed previously ${ }^{17}$. The mean somatic mutation rate across the TCGA cohort was 8.87 mutations per megabase $(\mathrm{Mb})$ of DNA (range: $0.5-48$, median: 5.78 ). The non-synonymous mutation rate was 6.86 per $\mathrm{Mb}$. MutSig2 $\mathrm{CV}^{18}$ identified significantly mutated genes among our 230 cases along with 182 similarly-sequenced, previously reported lung adenocarcinomas $^{12}$. Analysis of these 412 tumour/normal pairs highlighted 18 statistically significant mutated genes (Fig. 1a shows co-mutation plot of TCGA samples $(n=230)$, Supplementary Fig. 2 shows co-mutation plot of all samples used in the statistical analysis $(n=412)$ and Supplementary Table 4 contains complete MutSig2CV results, which also appear on the TCGA Data Portal along with many associated data files (https://tcga-data.nci.nih.gov/docs/publications/luad_2014/). TP53 was commonly mutated (46\%). Mutations in KRAS (33\%) were mutually exclusive with those in EGFR (14\%). BRAF was also commonly mutated (10\%), as were PIK3CA (7\%), MET (7\%) and the small GTPase gene, RIT1 (2\%). Mutations in tumour suppressor genes including STK11 (17\%), KEAP1 (17\%), NF1 (11\%), RB1 (4\%) and CDKN2A (4\%) were observed. Mutations in chromatin modifying genes SETD2 (9\%), ARID1A (7\%) and SMARCA4 (6\%) and the RNA splicing genes RBM10 (8\%) and U2AF1 (3\%) were also common. Recurrent mutations in the $M G A$ gene (which encodes a Max-interacting protein on the MYC pathway ${ }^{19}$ ) occurred in $8 \%$ of samples. Loss-of-function (frameshift and nonsense) mutations in MGA were mutually exclusive with focal MYC amplification (Fisher's exact test $P=0.04$ ), suggesting a hitherto unappreciated potential mechanism of MYC pathway activation. Coding single nucleotide variants and indel variants were verified by resequencing at a rate of $99 \%$ and $100 \%$, respectively (Supplementary Fig. 3a, Supplementary Table 5). Tumour purity was not associated with the presence of false negatives identified in the validation data $(P=0.31$; Supplementary Fig. $3 \mathrm{~b})$.

Past or present smoking associated with cytosine to adenine $(C>A)$ nucleotide transversions as previously described both in individual genes and genome-wide ${ }^{12,13} . \mathrm{C}>\mathrm{A}$ nucleotide transversion fraction showed two peaks; this fraction correlated with total mutation count $\left(R^{2}=0.30\right)$ and inversely correlated with cytosine to thymine $(C>T)$ transition frequency $\left(R^{2}=0.75\right)$ (Supplementary Fig. 4$)$. We classified each sample (Supplementary Methods) into one of two groups named transversionhigh (TH, $n=269$ ), and transversion-low (TL, $n=144)$. The transversionhigh group was strongly associated with past or present smoking $(P<$ $\left.2.2 \times 10^{-16}\right)$, consistent with previous reports ${ }^{13}$. The transversion-high and transversion-low patient cohorts harboured different gene mutations. Whereas KRAS mutations were significantly enriched in the transversionhigh cohort $\left(P=2.1 \times 10^{-13}\right)$, EGFR mutations were significantly enriched in the transversion-low group $\left(P=3.3 \times 10^{-6}\right)$. PIK3CA and $R B 1$ mutations were likewise enriched in transversion-low tumours $(P<0.05)$. Additionally, the transversion-low tumours were specifically enriched for in-frame insertions in EGFR and ERBB2 (ref. 5) and for frameshift indels in $R B 1$ (Fig. 1b). RB1 is commonly mutated in small-cell lung carcinoma (SCLC). We found RB1 mutations in transversion-low adenocarcinomas were enriched for frameshift indels versus single nucleotide substitutions compared to SCLC $(P<0.05)^{20,21}$ suggesting a mutational mechanism in transversion-low adenocarcinoma that is probably distinct from smoking in SCLC.

Gender is correlated with mutation patterns in lung adenocarcinoma ${ }^{22}$. Only a fraction of significantly mutated genes from the complete set reported in this study (Fig. 1a) were enriched in men or women (Fig. 1c). EGFR mutations were enriched in tumours from the female cohort $(P=0.03)$ whereas loss-of-function mutations within $R B M 10$, an RNA-binding protein located on the $\mathrm{X}$ chromosome ${ }^{23}$ were enriched in tumours from men $(P=0.002)$. When examining the transversion-high group, 16 out of 21 $R B M 10$ mutations were observed in males ( $P=0.003$, Fisher's exact test).

Somatic copy number alterations were very similar to those previously reported for lung adenocarcinoma ${ }^{24}$ (Supplementary Fig. 5, Supplementary Table 6). Significant amplifications included NKX2-1, TERT,
MDM2, KRAS, EGFR, MET, CCNE1, CCND1, TERC and MECOM (Supplementary Table 6), as previously described ${ }^{24}, 8 \mathrm{q} 24$ near $M Y C$, and a novel peak containing CCND3 (Supplementary Table 6). The CDKN2A locus was the most significant deletion (Supplementary Table 6). Supplementary Table 7 summarizes molecular and clinical characteristics by sample. Low-pass whole-genome sequencing on a subset $(n=93)$ of the samples revealed an average of 36 gene-gene and gene-inter-gene
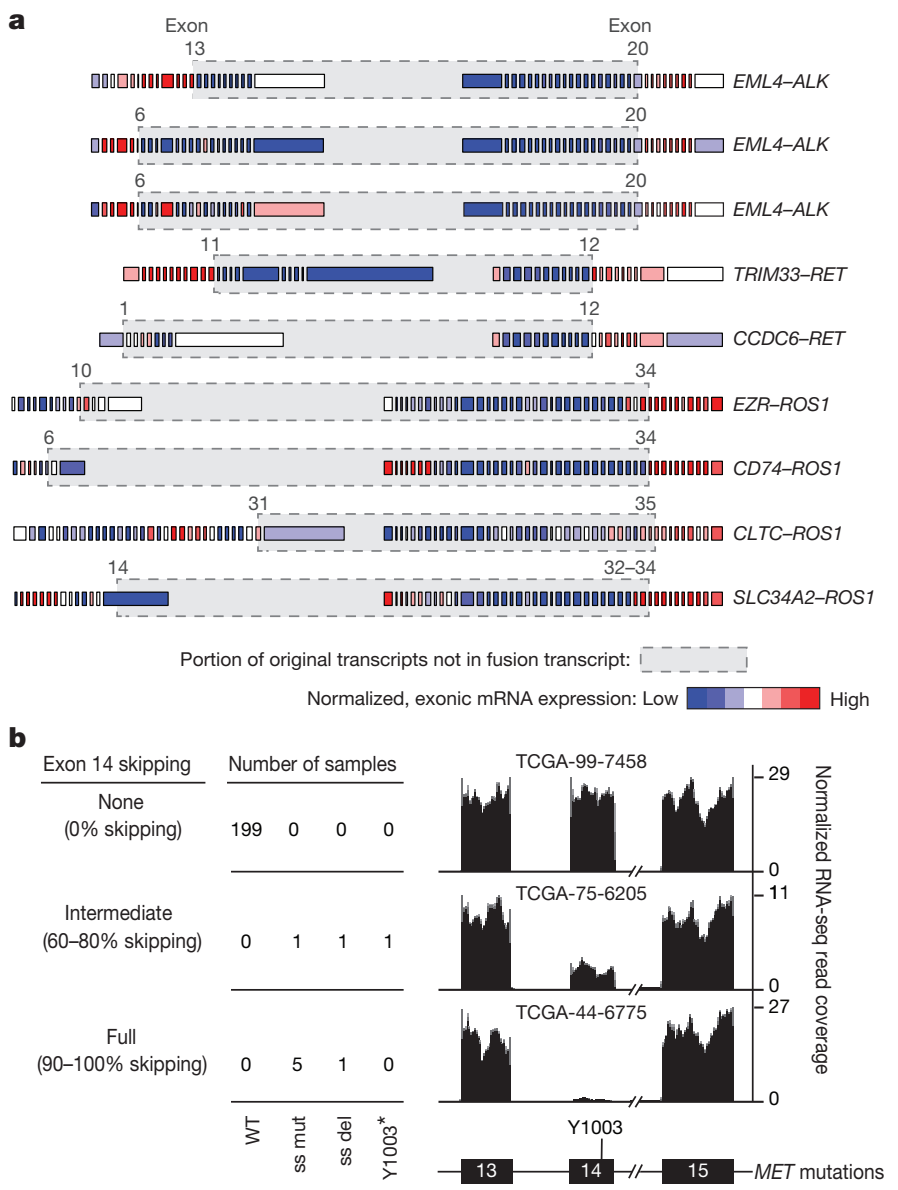

Observed splicing across all tumours (total events $=29,867$ )

Associated with U2AF1 S34F mutation (total events $=129 ; q$ value $<0.05$ )

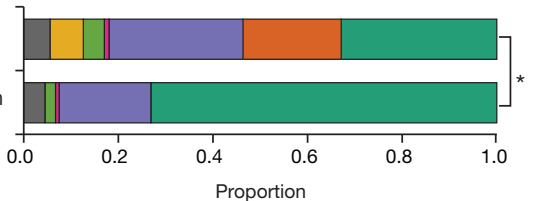
Cassette exon
$\overbrace{\text { Altenative }}^{\text {Casplice }}$
Coordinate cassette exons
Mutually exclusive exon
Alternative 5' splice site Alternative $3^{\prime}$ splice site Alternative first exon Alternative last exon

$$
\text { (1) Al }
$$

${ }^{\star} P<0.001$

Figure $2 \mid$ Aberrant RNA transcripts in lung adenocarcinoma associated with somatic DNA translocation or mutation. a, Normalized exon level RNA expression across fusion gene partners. Grey boxes around genes mark the regions that are removed as a consequence of the fusion. Junction points of the fusion events are also listed in Supplementary Table 9. Exon numbers refer to reference transcripts listed in Supplementary Table 9. b, MET exon 14 skipping observed in the presence of exon 14 splice site mutation (ss mut), splice site deletion (ss del) or a Y1003* mutation. A total of 22 samples had insufficient coverage around exon 14 for quantification. The percentage skipping is (total expression minus exon 14 expression)/total expression. c, Significant differences in the frequency of 129 alternative splicing events in mRNA from tumours with U2AF1 S34F tumours compared to U2AF1 WT tumours ( $q$ value $<0.05$ ). Consistent with the function of U2AF1 in $3^{\prime}$ splice site recognition, most splicing differences involved cassette exon and alternative $3^{\prime}$ splice site events (chi-squared test, $P<0.001$ ). 
rearrangements per tumour. Chromothripsis ${ }^{25}$ occurred in six of the 93 samples (6\%) (Supplementary Fig. 6, Supplementary Table 8). Lowpass whole genome sequencing-detected rearrangements appear in Supplementary Table 9 .

\section{Description of aberrant RNA transcripts}

Gene fusions, splice site mutations or mutations in genes encoding splicing factors promote or sustain the malignant phenotype by generating aberrant RNA transcripts. Combining DNA with mRNA sequencing enabled us to catalogue aberrant RNA transcripts and, in many cases, to identify the DNA-encoded mechanism for the aberration. Seventyfive per cent of somatic mutations identified by WES were present in the RNA transcriptome when the locus in question was expressed (minimum $5 \times)$ (Supplementary Fig. 7a) similar to prior analyses ${ }^{15}$. Previously identified fusions involving $A L K$ (3/230 cases), ROS1 (4/230) and RET $(2 / 230)$ (Fig. 2a, Supplementary Table 10), all occurred in transversionlow tumours $\left(P=1.85 \times 10^{-4}\right.$, Fisher's exact test $)$.

$M E T$ activation can occur by exon 14 skipping, which results in a stabilized protein ${ }^{26}$. Ten tumours had somatic MET DNA alterations with MET exon 14 skipping in RNA. In nine of these samples, a $5^{\prime}$ or $3^{\prime}$ splice site mutation or deletion was identified ${ }^{27}$. MET exon 14 skipping was also found in the setting of a MET Y1003* stop codon mutation (Fig. 2b, Supplementary Fig. 8a). The codon affected by the Y1003* mutation is predicted to disrupt multiple splicing enhancer sequences, but the mechanism of skipping remains unknown in this case.

S34F mutations in $U 2 A F 1$ have recently been reported in lung adenocarcinoma ${ }^{12}$ but their contribution to oncogenesis remains unknown. Eight samples harboured $U 2 A F 1^{\mathrm{S} 34 \mathrm{~F}}$. We identified 129 splicing events strongly associated with $U 2 A F 1^{\mathrm{S} 34 \mathrm{~F}}$ mutation, consistent with the role of U2AF1 in $3^{\prime}$-splice site selection ${ }^{28}$. Cassette exons and alternative $3^{\prime}$ splice sites were most commonly affected (Fig. 2c, Supplementary Table 11) ${ }^{29}$. Among these events, alternative splicing of the CTNNB1 proto-oncogene was strongly associated with $U 2 A F 1$ mutations (Supplementary Fig. 8b). Thus, concurrent analysis of DNA and RNA enabled delineation of both cis and trans mechanisms governing RNA processing in lung adenocarcinoma.

\section{Candidate driver genes}

The receptor tyrosine kinase (RTK)/RAS/RAF pathway is frequently mutated in lung adenocarcinoma. Striking therapeutic responses are often achieved when mutant pathway components are successfully inhibited. Sixty-two per cent (143/230) of tumours harboured known activating mutations in known driver oncogenes, as defined by others ${ }^{30}$. Cancerassociated mutations in $\operatorname{KRAS}(32 \%, n=74), E G F R(11 \%, n=26)$ and $B R A F(7 \%, n=16)$ were common. Additional, previously uncharacterized KRAS, EGFR and BRAF mutations were observed, but were not classified as driver oncogenes for the purposes of our analyses (see Supplementary Fig. 9a for depiction of all mutations of known and unknown significance); explaining the differing mutation frequencies in each gene between this analysis and the overall mutational analysis described above. We also identified known activating ERBB2 in-frame insertion and point mutations $(n=5)^{6}$, as well as mutations in MAP2K1 $(n=2), N R A S$ and $\operatorname{HRAS}(n=1$ each). RNA sequencing revealed the aforementioned MET exon 14 skipping $(n=10)$ and fusions involving ROS1 $(n=4), A L K$ $(n=3)$ and RET $(n=2)$. We considered these tumours collectively as oncogene-positive, as they harboured a known activating RTK/RAS/ RAF pathway somatic event. DNA amplification events were not considered to be driver events before the comparisons described below.

We sought to nominate previously unrecognized genomic events that might activate this critical pathway in the $38 \%$ of samples without a RTK/RAS/RAF oncogene mutation. Tumour cellularity did not differ between oncogene-negative and oncogene-positive samples (Supplementary Fig. 9b). Analysis of copy number alterations using GISTIC ${ }^{31}$ identified unique focal $E R B B 2$ and $M E T$ amplifications in the oncogene-negative subset (Fig. 3a, Supplementary Table 6); amplifications in other wild-type proto-oncogenes, including KRAS and EGFR, were not significantly different between the two groups.

We next analysed WES data independently in the oncogene-negative and oncogene-positive subsets. We found that TP53, KEAP1, NF1 and RIT1 mutations were significantly enriched in oncogene-negative tumours $(P<0.01$; Fig. 3b, Supplementary Table 12). NF1 mutations have previously been reported in lung adenocarcinoma ${ }^{11}$, but this is the first study, to our knowledge, capable of identifying all classes of loss-of-function a

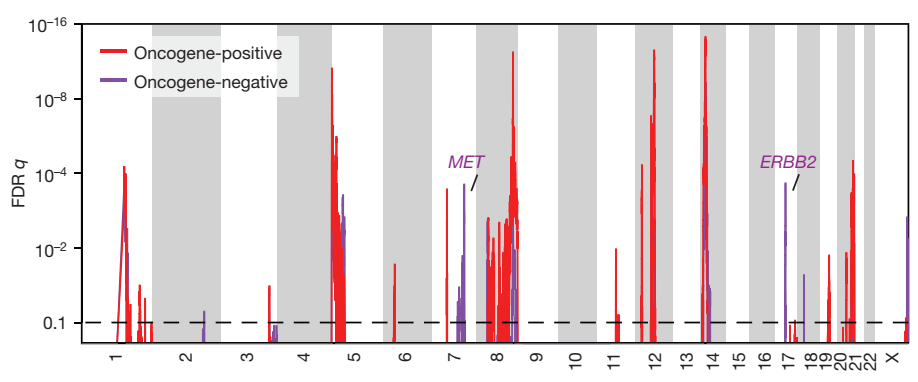

c

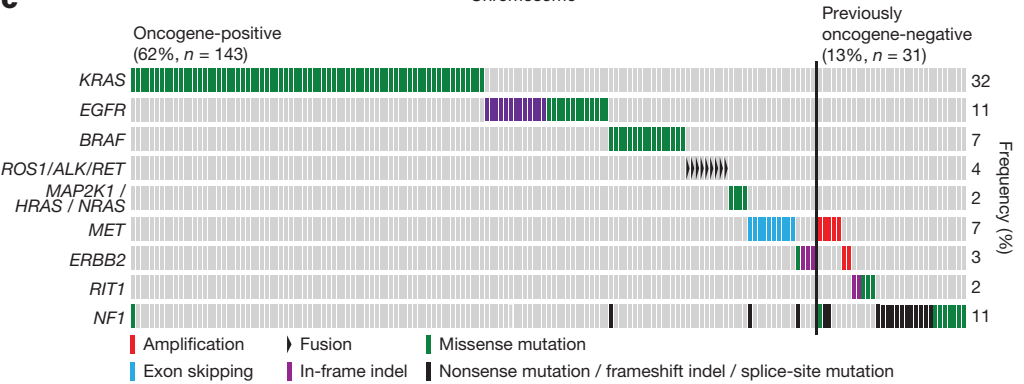

b

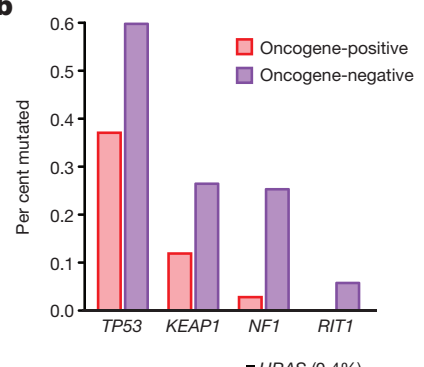

d

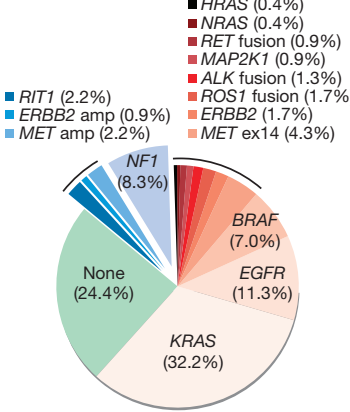

Figure 3 Identification of novel candidate driver genes. a, GISTIC analysis of focal amplifications in oncogene-negative $(n=87)$ and oncogene-positive $(n=143)$ TCGA samples identifies focal gains of MET and ERBB2 that are specific to the oncogene-negative set (purple). b, TP53, KEAP1, NF1 and RIT1 mutations are significantly enriched in samples otherwise lacking oncogene mutations (adjusted $P<0.05$ by Fisher's exact test). c, Co-mutation plot of variants of known significance within the RTK/RAS/RAF pathway in lung adenocarcinoma. Not shown are the 63 tumours lacking an identifiable driver lesion. Only canonical driver events, as defined in Supplementary Fig. 9, and proposed driver events, are shown; hence not every alteration found is displayed. d, New candidate driver oncogenes (blue: 13\% of cases) and known somatically activated drivers events (red: 63\%) that activate the RTK/RAS/RAF pathway can be found in the majority of the 230 lung adenocarcinomas. 
NF1 defects and to statistically demonstrate that NF1 mutations, as well as KEAP1 and TP53 mutations are enriched in the oncogene-negative subset of lung adenocarcinomas (Fig. 3c). All RIT1 mutations occurred in the oncogene-negative subset and clustered around residue Q79 (homologous to Q61 in the switch II region of RAS genes). These mutations transform NIH3T3 cells and activate MAPK and PI(3)K signalling ${ }^{32}$, supporting a driver role for mutant RIT1 in $2 \%$ of lung adenocarcinomas. This analysis increases the rate at which putative somatic lung adenocarcinoma driver events can be identified within the RTK/RAS/RAF pathway to $76 \%$ (Fig. 3d).

a
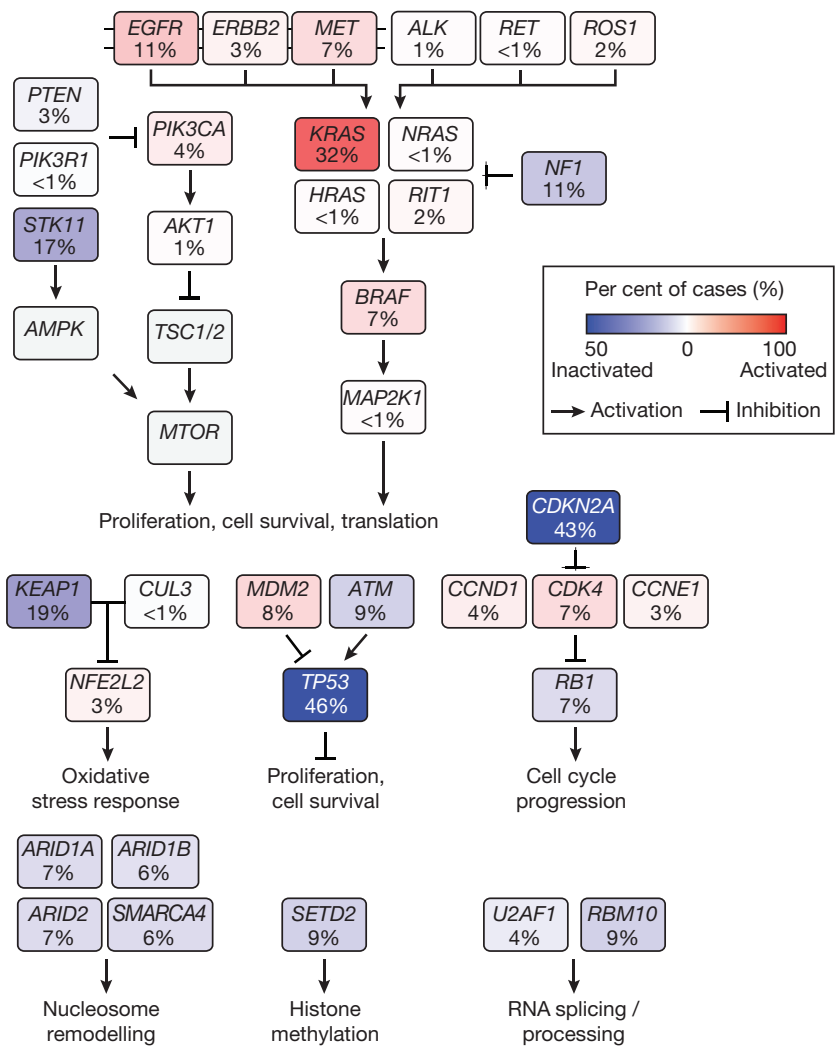

b MAPK pathway

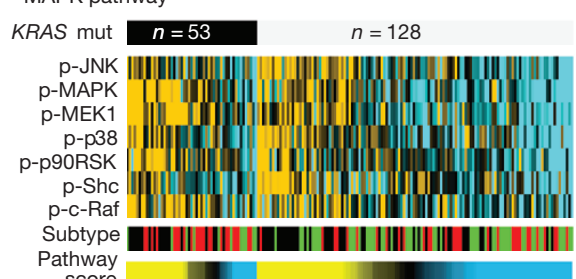

score

c $\mathrm{PI}(3) \mathrm{K}$ pathway

PIK3CA STK11 High Low

mut mut p-AKT p-AMPK Unaligned

$(n=9) \quad(n=42) \quad(n=35)(n=21) \quad(n=74)$
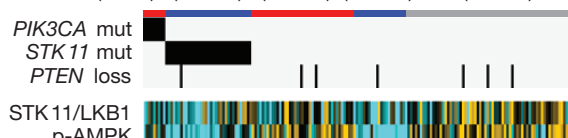

p-AMPK

p-mTOR

p-4E-BP1 p-p70S6K

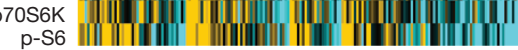

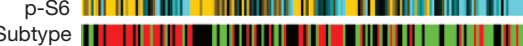

Pathway

score

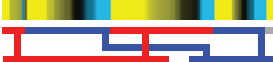

PI3K-Akt branch active LKB1-AMPK inactive

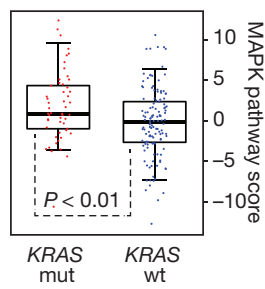

KRAS KRAS
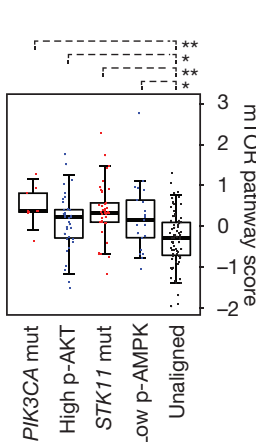

Protein expression

Expression subtype

Pathway signature

${ }^{*} P<0.01$

$\square$ Low $\square$ High $\square$ Low $\square$ High

${ }^{\star *} P<0.001$

\section{Recurrent alterations in key pathways}

Recurrent aberrations in multiple key pathways and processes characterize lung adenocarcinoma (Fig. 4a). Among these were RTK/RAS/ RAF pathway activation (76\% of cases), $\mathrm{PI}(3) \mathrm{K}-\mathrm{mTOR}$ pathway activation $(25 \%)$, p53 pathway alteration (63\%), alteration of cell cycle regulators (64\%, Supplementary Fig. 10), alteration of oxidative stress pathways (22\%, Supplementary Fig. 11), and mutation of various chromatin and RNA splicing factors (49\%).

We then examined the phenotypic sequelae of some key genomic events in the tumours in which they occurred. Reverse-phase protein arrays provided proteomic and phosphoproteomic phenotypic evidence of pathway activity. Antibodies on this platform are listed in Supplementary Table 13. This analysis suggested that DNA sequencing did not identify all samples with phosphoprotein evidence of activation of a given signalling pathway. For example, whereas $K R A S$-mutant lung adenocarcinomas had higher levels of phosphorylated MAPK than KRAS wild-type tumours had on average, many KRAS wild-type tumours displayed significant MAPK pathway activation (Fig. 4b, Supplementary Fig. 10). The multiple mechanisms by which lung adenocarcinomas achieve MAPK activation suggest additional, still undetected RTK/RAS/ RAF pathway alterations. Similarly, we found significant activation of $\mathrm{mTOR}$ and its effectors (p70S6kinase, S6, 4E-BP1) in a substantial fraction of the tumours (Fig. 4c). Analysis of mutations in PIK3CA and STK11, STK11 protein levels, and AMPK and AKT phosphorylation ${ }^{33}$ led to the identification of three major mTOR patterns in lung adenocarcinoma: (1) tumours with minimal or basal mTOR pathway activation, (2) tumours showing higher mTOR activity accompanied by either STK11-inactivating mutation or combined low STK11 expression and low AMPK activation and (3) tumours showing high mTOR activity accompanied by either phosphorylated AKT activation, PIK3CA mutation, or both. As with MAPK, many tumours lack an obvious underlying genomic alteration to explain their apparent mTOR activation.

\section{Molecular subtypes of lung adenocarcinoma}

Broad transcriptional and epigenetic profiling can reveal downstream consequences of driver mutations, provide clinically relevant classification and offer insight into tumours lacking clear drivers. Prior unsupervised analyses of lung adenocarcinoma gene expression have used varying nomenclature for transcriptional subtypes of the disease ${ }^{34-37}$. To coordinate naming of the transcriptional subtypes with the histopathological ${ }^{38}$, anatomic and mutational classifications of lung adenocarcinoma, we propose an updated nomenclature: the terminal respiratory unit (TRU, formerly bronchioid), the proximal-inflammatory (PI, formerly squamoid), and the proximal-proliferative (PP, formerly magnoid) ${ }^{39}$ transcriptional subtypes (Fig. 5a). Previously reported associations of expression signatures with pathways and clinical outcomes ${ }^{34,36,39}$ were observed (Supplementary Fig. 7b) and integration with multi-analyte data revealed statistically significant genomic alterations associated with these transcriptional subtypes. The PP subtype was enriched for mutation of KRAS, along with inactivation of the STK11 tumour suppressor gene by chromosomal loss, inactivating mutation, and reduced gene expression. In contrast, the PI subtype was characterized by solid histopathology and

Figure 4 | Pathway alterations in lung adenocarcinoma. a, Somatic alterations involving key pathway components for RTK signalling, mTOR signalling, oxidative stress response, proliferation and cell cycle progression, nucleosome remodelling, histone methylation, and RNA splicing/processing. b, c, Proteomic analysis by RPPA $(n=181) P$ values by two-sided $t$-test. Box plots represent 5\%, 25\%, 75\%, median, and 95\%. PP, proximal proliferative; TRU, terminal respiratory unit; PI, proximal inflammatory. c, mTOR signalling may be activated, by either Akt (for example, via PI(3)K) or inactivation of AMPK (for example, via STK11 loss). Tumours were separated into three main groups: those with $\mathrm{PI}(3) \mathrm{K}-\mathrm{AKT}$ activation, through either PIK3CA activating mutation or unknown mechanism (high p-AKT); those with LKB1-AMPK inactivation, through either STK11 mutation or unknown mechanism with low levels of LKB1 and p-AMPK; and those showing none of the above features. 
C Integrated subtypes

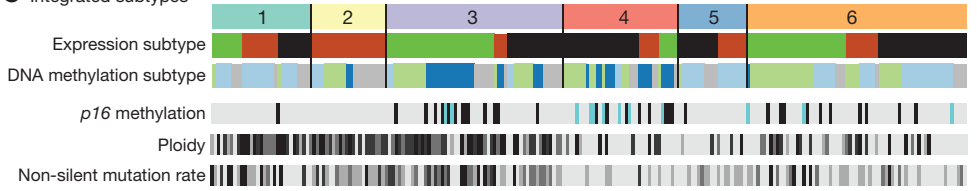

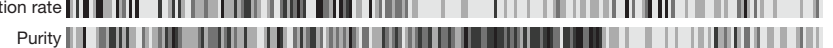

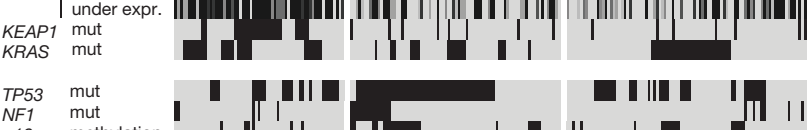

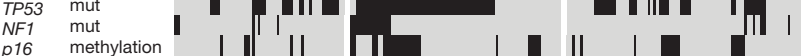

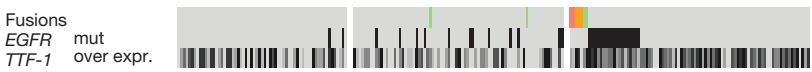

Mutation total

Ploidy

$\stackrel{\text { Purity }}{\mathrm{CpG} \rightarrow \mathrm{T} \%}$

Never-smoker

Female

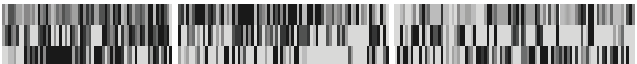

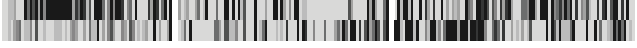

Histology
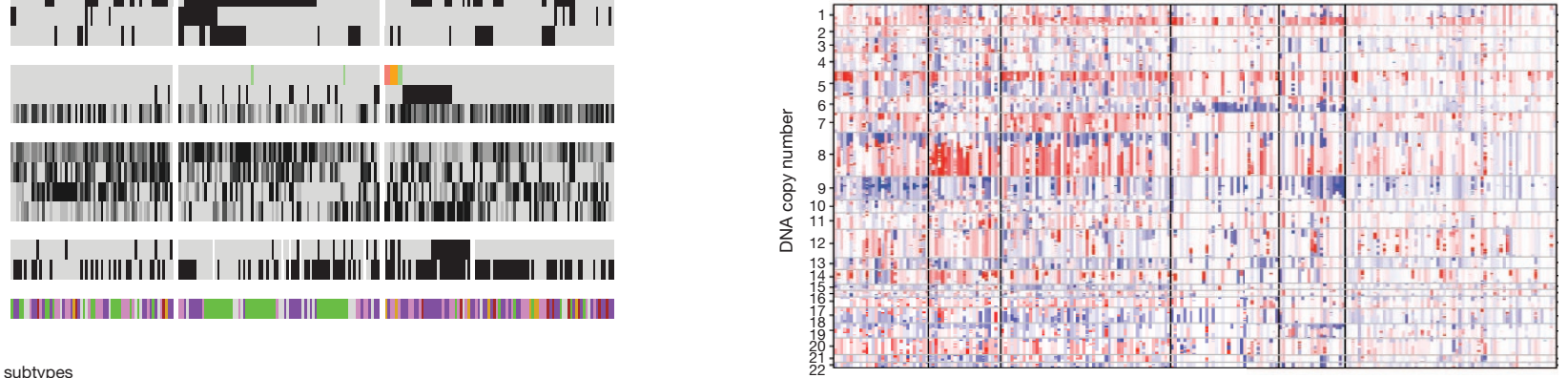

b DNA methylation subtypes
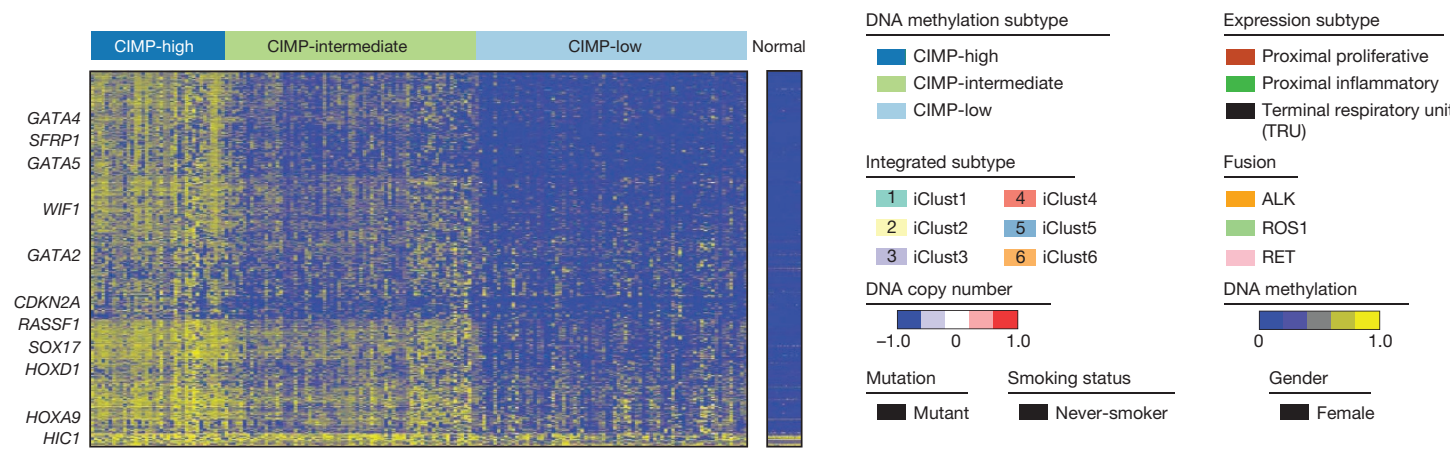

Expression, ploidy, purity, mutation rates

CIMP-high

CIMP-intermediat

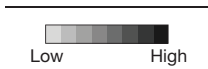

tegrated subtype

DA copy number

$0 \quad 1.0$

Mutant

Never-smoker

Female

\section{Histology}

Solid

Acinar

Lepidic

Papillary/Micropapillary

Mucinous

Other

Figure 5 | Integrative analysis. a-c, Integrating unsupervised analyses of 230 lung adenocarcinomas reveals significant interactions between molecular subtypes. Tumours are displayed as columns, grouped by mRNA expression subtypes (a), DNA methylation subtypes (b), and integrated subtypes by

co-mutation of NF1 and TP53. Finally, the TRU subtype harboured the majority of the EGFR-mutated tumours as well as the kinase fusion expressing tumours. TRU subtype membership was prognostically favourable, as seen previously ${ }^{34}$ (Supplementary Fig. 7c). Finally, the subtypes exhibited different mutation rates, transition frequencies, genomic ploidy profiles, patterns of large-scale aberration, and differed in their association with smoking history (Fig. 5a). Unsupervised clustering of miRNA sequencing-derived or reverse phase protein array (RPPA)-derived data also revealed significant heterogeneity, partially overlapping with the mRNA-based subtypes, as demonstrated in Supplementary Figs 12 and 13.

Mutations in chromatin-modifying genes (for example, SMARCA4, $A R I D 1 A$ and SETD2) suggest a major role for chromatin maintenance in lung adenocarcinoma. To examine chromatin states in an unbiased manner, we selected the most variable DNA methylation-specific probes in CpG island promoter regions and clustered them by methylation intensity (Supplementary Table 14). This analysis divided samples into two distinct subsets: a significantly altered $\mathrm{CpG}$ island methylator phenotypehigh (CIMP-H(igh)) cluster and a more normal-like CIMP-L(ow) group, with a third set of samples occupying an intermediate level of methylation at CIMP sites (Fig. 5b). Our results confirm a prior report ${ }^{40}$ and provide additional insights into this epigenetic program. CIMP-H tumours often showed DNA hypermethylation of several key genes: CDKN2A, GATA2, GATA4, GATA5, HIC1, HOXA9, HOXD13, RASSF1, SFRP1, SOX17 and WIF1 among others (Supplementary Fig. 14). WNT pathway genes are significantly over-represented in this list $(P$ value $=0.0015)$ suggesting that this is a key pathway with an important driving role within this subtype. MYC overexpression was significantly associated with the CIMP-H phenotype as well $(P=0.003)$.

Although we did not find significant correlations between global DNA methylation patterns and individual mutations in chromatin remodelling genes, there was an intriguing association between SETD2 mutation

iCluster analysis (c). All displayed features are significantly associated with subtypes depicted. The CIMP phenotype is defined by the most variable CpG island and promoter probes.

and $C D K N 2 A$ methylation. Tumours with low CDKN2A expression due to methylation (rather than due to mutation or deletion) had lower ploidy, fewer overall mutations (Fig. 5c) and were significantly enriched for SETD2 mutation, suggesting an important role for this chromatinmodifying gene in the development of certain tumours.

Integrative clustering ${ }^{41}$ of copy number, DNA methylation and mRNA expression data found six clusters (Fig. 5c). Tumour ploidy and mutation rate are higher in clusters $1-3$ than in clusters $4-6$. Clusters $1-3$ frequently harbour TP53 mutations and are enriched for the two proximal transcriptional subtypes. Fisher's combined probability tests revealed significant copy number associated gene expression changes on $3 q$ in cluster one, $8 \mathrm{q}$ in cluster two, and chromosome 7 and $15 q$ in cluster three (Supplementary Fig. 15). The low ploidy and low mutation rate clusters four and five contain many TRU samples, whereas tumours in cluster 6 have comparatively lower tumour cellularity, and few other distinguishing molecular features. Significant copy number-associated gene expression changes are observed on $6 \mathrm{q}$ in cluster four and $19 \mathrm{p}$ in cluster five. The CIMP-H tumours divided into a high ploidy, high mutation rate, proximal-inflammatory CIMP-H group (cluster 3) and a low ploidy, low mutation rate, TRU-associated CIMP-H group (cluster 4), suggesting that the CIMP phenotype in lung adenocarcinoma can occur in markedly different genomic and transcriptional contexts. Furthermore, cluster four is enriched for CDKN2A methylation and SETD2 mutations, suggesting an interaction between somatic mutation of SETD2 and deregulated chromatin maintenance in this subtype. Finally, cluster membership was significantly associated with mutations in TP53, EGFR and STK11 (Supplementary Fig. 15, Supplementary Table 6).

\section{Conclusions}

We assessed the mutation profiles, structural rearrangements, copy number alterations, DNA methylation, mRNA, miRNA and protein expression 
of 230 lung adenocarcinomas. In recent years, the treatment of lung adenocarcinoma has been advanced by the development of multiple therapies targeted against alterations in the RTK/RAS/RAF pathway. We nominate amplifications in MET and ERBB2 as well as mutations of NF1 and RIT1 as driver events specifically in otherwise oncogene-negative lung adenocarcinomas. This analysis increases the fraction of lung adenocarcinoma cases with somatic evidence of RTK/RAS/RAF activation from $62 \%$ to $76 \%$. While all lung adenocarcinomas may activate this pathway by some mechanism, only a subset show tonic pathway activation at the protein level, suggesting both diversity between tumours with seemingly similar activating events and as yet undescribed mechanisms of pathway activation. Therefore, the current study expands the range of possible targetable alterations within the RTK/RAS/RAF pathway in general and suggests increased implementation of MET and ERBB2/HER2 inhibitors in particular. Our discovery of inactivating mutations of $M G A$ further underscores the importance of the MYC pathway in lung adenocarcinoma.

This study further implicates both chromatin modifications and splicing alterations in lung adenocarcinoma through the integration of DNA, transcriptome and methylome analysis. We identified alternative splicing due to both splicing factor mutations in trans and mutation of splice sites in cis, the latter leading to activation of the MET gene by exon 14 skipping. Cluster analysis separated tumours based on single-gene driver events as well as large-scale aberrations, emphasizing lung adenocarcinoma's molecular heterogeneity and combinatorial alterations, including the identification of coincident SETD2 mutations and CDKN2A methylation in a subset of CIMP-H tumours, providing evidence of a somatic event associated with a genome-wide methylation phenotype. These studies provide new knowledge by illuminating modes of genomic alteration, highlighting previously unappreciated altered genes, and enabling further refinement in sub-classification for the improved personalization of treatment for this deadly disease.

\section{METHODS SUMMARY}

All specimens were obtained from patients with appropriate consent from the relevant institutional review board. DNA and RNA were collected from samples using the Allprep kit (Qiagen). We used standard approaches for capture and sequencing of exomes from tumour DNA and normal DNA ${ }^{15}$ and whole-genome shotgun sequencing. Significantly mutated genes were identified by comparing them with expectation models based on the exact measured rates of specific sequence lesions ${ }^{42}$. GISTIC analysis of the circular-binary-segmented Affymetrix SNP 6.0 copy number data was used to identify recurrent amplification and deletion peaks ${ }^{31}$. Consensus clustering approaches were used to analyse mRNA, miRNA and methylation subtypes using previous approaches ${ }^{15}$. The publication web page is (https://tcga-data.nci.nih.gov/ docs/publications/luad_2014/). Sequence files are in CGHub (https://cghub.ucsc.edu/).

Received 11 June 2013; accepted 22 April 2014.

Published online 9 July 2014.

1. Paez, J.G. etal. EGFR mutations in lung cancer: correlation with clinical response to gefitinib therapy. Science 304, 1497-1500 (2004).

2. Kwak, E. L. et al. Anaplastic lymphoma kinase inhibition in non-small-cell lung cancer. N. Engl. J. Med. 363, 1693-1703 (2010).

3. Bergethon, K. et al. ROS1 rearrangements define a unique molecular class of lung cancers. J. Clin Oncol. 30, 863-870 (2012).

4. Drilon, A. et al. Response to cabozantinib in patients with RET fusion-positive lung adenocarcinomas. Cancer Discov. 3, 630-635 (2013).

5. Stephens, P. et al. Lung cancer: intragenic ERBB2 kinase mutations in tumours. Nature 431, 525-526 (2004)

6. Takahashi, T. et al. p53: a frequent target for genetic abnormalities in lung cancer. Science 246, 491-494 (1989)

7. Sanchez-Cespedes, M. et al. Inactivation of LKB1/STK11 is a common event in adenocarcinomas of the lung. Cancer Res. 62, 3659-3662 (2002).

8. Shapiro, G. I. et al. Reciprocal Rb inactivation and $\mathrm{p} 16^{\mathrm{INK} 4}$ expression in primary lung cancers and cell lines. Cancer Res. 55, 505-509 (1995).

9. Singh, A. et al. Dysfunctional KEAP1-NRF2 interaction in non-small-cell lung cancer. PLoS Med. 3, e420 (2006)

10. Medina, P. P. et al. Frequent BRG1/SMARCA4-inactivating mutations in human lung cancer cell lines. Hum. Mutat. 29, 617-622 (2008).

11. Ding, L. et al. Somatic mutations affect key pathways in lung adenocarcinoma. Nature 455, 1069-1075 (2008)

12. Imielinski, M. etal. Mapping the hallmarks of lung adenocarcinoma with massively parallel sequencing. Cell 150, 1107-1120 (2012).
13. Govindan, R. et al. Genomic landscape of non-small cell lung cancer in smokers and never-smokers. Cell 150, 1121-1134 (2012).

14. Travis, W. D., Brambilla, E. \& Riely, G. J. New pathologic classification of lung cancer: relevance for clinical practice and clinical trials. J. Clin. Oncol. 31 , 992-1001 (2013).

15. The Cancer Genome Atlas Research Network Comprehensive genomic characterization of squamous cell lung cancers. Nature 489, 519-525 (2012).

16. Carter, S. L. et al. Absolute quantification of somatic DNA alterations in human cancer. Nature Biotechnol. 30, 413-421 (2012).

17. Cibulskis, K. et al. Sensitive detection of somatic point mutations in impure and heterogeneous cancer samples. Nature Biotechnol. 31, 213-219 (2013).

18. Lawrence, M. S. et al. Discovery and saturation analysis of cancer genes across 21 tumour types. Nature 505, 495-501 (2014).

19. Hurlin, P. J., Steingrimsson, E., Copeland, N. G., Jenkins, N. A. \& Eisenman, R. N. Mga, a dual-specificity transcription factor that interacts with Max and contains a T-domain DNA-binding motif. EMBO J. 18, 7019-7028 (1999).

20. Peifer, M. et al. Integrative genome analyses identify key somatic driver mutations of small-cell lung cancer. Nature Genet. 44, 1104-1110 (2012).

21. Rudin, C. M. et al. Comprehensive genomic analysis identifies SOX2 as a frequently amplified gene in small-cell lung cancer. Nature Genet. 44, 1111-1116 (2012).

22. Tokumo, M. et al. The relationship between epidermal growth factor receptor mutations and clinicopathologic features in non-small cell lung cancers. Clin. Cancer Res. 11, 1167-1173 (2005)

23. Coleman, M. P. et al. A novel gene, DXS8237E, lies within $20 \mathrm{~kb}$ upstream of UBE in Xp11.23 and has a different X inactivation status. Genomics 31, 135-138 (1996).

24. Weir, B. A. et al. Characterizing the cancer genome in lung adenocarcinoma. Nature 450, 893-898 (2007)

25. Stephens, P. J. et al. Massive genomic rearrangement acquired in a single catastrophic event during cancer development. Cell 144, 27-40 (2011)

26. Kong-Beltran, M. et al. Somatic mutations lead to an oncogenic deletion of Met in lung cancer. Cancer Res. 66, 283-289 (2006).

27. Seo, J. S. et al. The transcriptional landscape and mutational profile of lung adenocarcinoma. Genome Res. 22, 2109-2119 (2012)

28. Wu, S., Romfo, C. M., Nilsen, T. W. \& Green, M. R. Functional recognition of the $3^{\prime}$ splice site $A G$ by the splicing factor $U 2 A F^{35}$. Nature $402,832-835$ (1999).

29. Brooks, A. N. etal. A pan-cancer analysis of transcriptome changes associated with somatic mutations in U2AF1 reveals commonly altered splicing events. PLOS ONE 9, e87361 (2014)

30. Pao, W. \& Hutchinson, K. E. Chipping away at the lung cancer genome. Nature Med. 18, 349-351 (2012).

31. Beroukhim, R. et al. Assessing the significance of chromosomal aberrations in cancer: methodology and application to glioma. Proc. Natl Acad. Sci. USA 104 20007-20012 (2007).

32. Berger, A. H. et al. Oncogenic RIT1 mutations in lung adenocarcinoma. Oncogene http://dx.doi.org/10.1038/onc.2013.581 (2014).

33. Creighton, C. J. et al. Proteomic and transcriptomic profiling reveals a link between the PI3K pathway and lower estrogen-receptor (ER) levels and activity in $\mathrm{ER}^{+}$ breast cancer. Breast Cancer Res. 12, R40 (2010).

34. Wilkerson, M. D. et al. Differential pathogenesis of lung adenocarcinoma subtypes involving sequence mutations, copy number, chromosomal instability, and methylation. PLOS ONE 7, e36530 (2012).

35. Beer, D. G. et al. Gene-expression profiles predict survival of patients with lung adenocarcinoma. Nature Med. 8, 816-824 (2002)

36. Hayes, D. N. et al. Gene expression profiling reveals reproducible human lung adenocarcinoma subtypes in multiple independent patient cohorts. J. Clin. Oncol. 24, 5079-5090 (2006)

37. Bhattacharjee, A. et al. Classification of human lung carcinomas by mRNA expression profiling reveals distinct adenocarcinoma subclasses. Proc. Natl Acad. Sci. USA 98, 13790-13795 (2001).

38. Travis, W. D. et al. International association for the study of lung cancer/American Thoracic Society/European Respiratory Society international multidisciplinary classification of lung adenocarcinoma. J. Thoracic Oncol. 6, 244-285 (2011).

39. Yatabe, Y., Mitsudomi, T.\& Takahashi, T. TTF-1 expression in pulmonary adenocarcinomas. Am. J. Surg. Pathol. 26, 767-773 (2002)

40. Shinjo, K. et al. Integrated analysis of genetic and epigenetic alterations reveals $\mathrm{CpG}$ island methylator phenotype associated with distinct clinical characters of lung adenocarcinoma. Carcinogenesis 33, 1277-1285 (2012)

41. Mo, Q. et al. Pattern discovery and cancer gene identification in integrated cancer genomic data. Proc. Natl Acad. Sci. USA 110, 4245-4250 (2013).

42. Lawrence, M. S. et al. Mutational heterogeneity in cancer and the search for new cancer-associated genes. Nature 499, 214-218 (2013)

Supplementary Information is available in the online version of the paper

Acknowledgements This study was supported by NIH grants: U24 CA126561, U24 CA126551, U24 CA126554, U24 CA126543, U24 CA126546, U24 CA137153, U24 CA126563, U24 CA126544, U24 CA143845, U24 CA143858, U24 CA144025, U24 CA143882, U24 CA143866, U24 CA143867, U24 CA143848, U24 CA143840, U24 CA143835, U24 CA143799, U24 CA143883, U24 CA143843, U54 HG003067, U54 HG003079 and U54 HG003273. We thank K. Guebert and L. Gaffney for assistance and C. Gunter for review. 
Author Contributions The Cancer Genome Atlas Research Network contributed collectively to this study. Biospecimens were provided by the tissue source sites and processed by the biospecimen core resource. Data generation and analyses were performed by the genome sequencing centres, cancer genome characterization centres and genome data analysis centres. All data were released through the data coordinating centre. The National Cancer Institute and National Human Genome Research Institute project teams coordinated project activities. We also acknowledge the following TCGA investigators who made substantial contributions to the project: E. A. Collisson (manuscript coordinator); J. D. Campbell, J. Chmielecki, (analysis coordinators); C. Sougnez (data coordinator); J. D. Campbell, M. Rosenberg, W. Lee J. Chmielecki, M. Ladanyi, and G. Getz (DNA sequence analysis); M. D. Wilkerson, A. N. Brooks, and D. N. Hayes (mRNA sequence analysis); L. Danilova and L. Cope (DNA methylation analysis); A. D. Cherniack (copy number analysis); M. D. Wilkerson and A. Hadjipanayis (translocations); N. Schultz, W. Lee, E. A. Collisson, A. H. Berger, J. Chmielecki, C. J. Creighton, L. A. Byers and M. Ladanyi (pathway analysis); A. Chu and A. G. Robertson (miRNA sequence analysis); W. Travis and D. A. Wigle (pathology and clinical expertise); L. A. Byers and G. B. Mills (reverse phase protein arrays); S. B. Baylin, R. Govindan and M. Meyerson (project chairs)

Author Information The primary and processed data used to generate the analyses presented here can be downloaded by registered users from The Cancer Genome Atlas at (https://tcga-data.nci.nih.gov/tcga/tcgaDownload.jsp). All of the primary sequence files are deposited in cgHub and all other data are deposited at the Data Coordinating Center (DCC) for public access (http://cancergenome.nih.gov/), (https://

cghub.ucsc.edu/) and (https://tcga-data.nci.nih.gov/docs/publications/luad_2014/). Reprints and permissions information is available at www.nature.com/reprints. The authors declare no competing financial interests. Readers are welcome to comment on the online version of the paper. Correspondence and requests for materials should be addressed to M.M. (matthew_meyerson@dfci.harvard.edu).

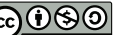

This work is licensed under a Creative Commons Attribution-

NonCommercial-ShareAlike 3.0 Unported licence. The images or other third party material in this article are included in the article's Creative Commons licence unless indicated otherwise in the credit line; if the material is not included under the Creative Commons licence, users will need to obtain permission from the licence holder to reproduce the material. To view a copy of this licence, visit http://creativecommons. org/licenses/by-nc-sa/3.0

\section{The Cancer Genome Atlas Research Network}

Disease analysis working group Eric A. Collisson ${ }^{1}$, Joshua D. Campbell ${ }^{2}$, Angela N. Brooks $^{2,3}$, Alice H. Berger ${ }^{2}$, William Lee ${ }^{4}$, Juliann Chmielecki ${ }^{2}$, David G. Beer ${ }^{5}$, Leslie Cope $^{6}$, Chad J. Creighton7", Ludmila Danilova ${ }^{6}$, Li Ding ${ }^{8}$, Gad Getz $^{2,9,10}$, Peter S. Hammerman $^{2}$, D. Neil Hayes ${ }^{11}$, Bryan Hernandez ${ }^{2}$, James G. Herman ${ }^{6}$, John V. Heymach $^{12}$, Igor Jurisica ${ }^{13}$, Raju Kucherlapati ${ }^{9}$, David Kwiatkowski ${ }^{14}$, Marc Ladanyi ${ }^{4}$, Gordon Robertson ${ }^{15}$, Nikolaus Schultz ${ }^{4}$, Ronglai Shen ${ }^{4}$, Rileen Sinha ${ }^{12}$ Carrie Sougnez ${ }^{2}$, Ming-Sound Tsao ${ }^{13}$, William D. Travis ${ }^{4}$, John N. Weinstein ${ }^{12}$, Dennis A. Wigle ${ }^{16}$, Matthew D. Wilkerson ${ }^{11}$, Andy Chu ${ }^{15}$, Andrew D. Cherniack ${ }^{2}$, Angela Hadjipanayis ${ }^{9}$, Mara Rosenberg ${ }^{2}$, Daniel J. Weisenberger ${ }^{17}$, Peter W. Laird ${ }^{17}$, Amie Radenbaugh ${ }^{18}$, Singer Ma ${ }^{18}$, Joshua M. Stuart ${ }^{18}$, Lauren Averett Byers ${ }^{12}$, Stephen B. Baylin ${ }^{6}$, Ramaswamy Govindan ${ }^{8}$, Matthew Meyerson ${ }^{2,3}$

Genome sequencing centres: The Eli \& Edythe L. Broad Institute Mara Rosenberg ${ }^{2}$, Stacey B. Gabriel ${ }^{2}$, Kristian Cibulskis ${ }^{2}$, Carrie Sougnez ${ }^{2}$, Jaegil Kim ${ }^{2}$, Chip Stewart ${ }^{2}$, Lee Lichtenstein ${ }^{2}$, Eric S. Lander ${ }^{2,19}$, Michael S. Lawrence ${ }^{2}$, Getz $^{2,9,10}$; Washington University in St. Louis Cyriac Kandoth ${ }^{8}$, Robert Fulton ${ }^{8}$, Lucinda L. Fulton ${ }^{8}$, Michael D. McLellan ${ }^{8}$, Richard K. Wilson ${ }^{8}$, Kai Ye ${ }^{8}$, Catrina C. Fronick ${ }^{8}$, Christopher A. Maher ${ }^{8}$, Christopher A. Miller ${ }^{8}$, Michael C. Wendl ${ }^{8}$, Christopher Cabanski ${ }^{8}$, Li Ding, Elaine Mardis $^{8}$, Ramaswamy Govindan ${ }^{8}$; Baylor College of Medicine Chad J. Creighton?, David Wheeler ${ }^{7}$

Genome characterization centres: Canada's Michael Smith Genome Sciences Centre, British Columbia Cancer Agency Miruna Balasundaram ${ }^{15}$, Yaron S. N. Butterfield ${ }^{15}$, Rebecca Carlsen ${ }^{15}$, Andy Chu ${ }^{15}$, Eric Chuah ${ }^{15}$, Noreen Dhalla ${ }^{15}$, Ranabir Guin ${ }^{15}$. Carrie Hirst ${ }^{15}$, Darlene Lee ${ }^{15}$, Haiyan I. Li ${ }^{15}$, Michael Mayo ${ }^{15}$, Richard A Moore ${ }^{15}$, Andrew J. Mungall ${ }^{15}$, Jacqueline E. Schein ${ }^{15}$, Payal Sipahimalani ${ }^{15}$, Angela Tam $^{15}$, Richard Varhol ${ }^{15}$, A. Gordon Robertson ${ }^{15}$, Natasja Wye ${ }^{15}$, Nina Thiessen ${ }^{15}$, Robert A. Holt ${ }^{12}$, Steven J. M. Jones ${ }^{15}$, Marco A. Marra ${ }^{15}$; The Eli \& Edythe L. Broad Institute Joshua D. Campbell ${ }^{2}$, Angela N. Brooks ${ }^{2,3}$, Juliann Chmielecki ${ }^{2}$, Marcin Imielinski ${ }^{2,9,10}$, Robert C. Onofrio ${ }^{2}$, Eran Hodis ${ }^{9}$, Travis Zack ${ }^{2}$, Carrie Sougnez ${ }^{2}$ Elena Helman ${ }^{2}$, Chandra Sekhar Pedamallu², Jill Mesirov², Andrew D. Cherniack ${ }^{2}$, Gordon Saksena ${ }^{2}$, Steven E. Schumacher ${ }^{2}$, Scott L. Carter ${ }^{2}$, Bryan Hernandez ${ }^{2}$, Levi Garraway $^{2,3,9}$, Rameen Beroukhim ${ }^{2,3,9}$, Stacey B. Gabriel ${ }^{2}$, Gad Getz ${ }^{2,9,10}$, Matthew Meyerson $^{2,3,9}$; Harvard Medical School/Brigham \& Women's Hospital/MD Anderson Cancer Center Angela Hadjipanayis ${ }^{9,14}$, Semin Lee L,14 $^{\text {, Harshad S. Mahadeshwar }}{ }^{12}$ Angeliki Pantazi ${ }^{9,14}$, Alexei Protopopov ${ }^{12}$, Xiaojia Ren $^{9}$, Sahil Seth ${ }^{12}$, Xingzhi Song ${ }^{12}$, Jiabin Tang ${ }^{12}$, Lixing Yang ${ }^{9}$, Jianhua Zhang ${ }^{12}$, Peng-Chieh Chen ${ }^{9}$, Michael Parfenov ${ }^{9}, 1^{\prime 4}$ Andrew Wei Xu ${ }^{9,14}$, Netty Santoso ${ }^{9,14}$, Lynda Chin ${ }^{12}$, Peter J. Park ${ }^{9,14}$ \& Raju Kucherlapati ${ }^{9,14}$; University of North Carolina, Chapel Hill Katherine A. Hoadley ${ }^{11}$, J. Todd Auman ${ }^{11}$, Shaowu Meng ${ }^{11}$, Yan Shi ${ }^{11}$, Elizabeth Buda ${ }^{11}$, Scot Waring ${ }^{11}$ Umadevi Veluvolu ${ }^{11}$, Donghui Tan ${ }^{11}$, Piotr A. Mieczkowski ${ }^{11}$, Corbin D. Jones ${ }^{11}$, Janae V. Simons ${ }^{11}$, Matthew G. Soloway ${ }^{11}$, Tom Bodenheimer ${ }^{11}$, Stuart R. Jefferys ${ }^{11}$, Jeffrey Roach $^{11}$, Alan P. Hoyle ${ }^{11}$, Junyuan Wu ${ }^{11}$, Saianand Balu ${ }^{11}$, Darshan Singh ${ }^{11}$, Jan F.
Prins $^{11}$, J.S. Marron ${ }^{11}$, Joel S. Parker ${ }^{11}$, D. Neil Hayes ${ }^{11}$, Charles M. Perou ${ }^{11}$; University of Kentucky Jinze Liu ${ }^{20}$; The USC/JHU Epigenome Characterization Center Leslie $\mathrm{Cope}^{6}$, Ludmila Danilova ${ }^{6}$, Daniel J. Weisenberger ${ }^{17}$, Dennis T. Maglinte ${ }^{17}$, Philip H. Lai $^{17}$, Moiz S. Bootwalla ${ }^{17}$, David J. Van Den Berg ${ }^{17}$, Timothy Triche Jr ${ }^{17}$, Stephen B. Baylin $^{6}$, Peter W. Laird ${ }^{17}$

Genome data analysis centres: The Eli \& Edythe L. Broad Institute Mara Rosenberg ${ }^{2}$ Lynda Chin ${ }^{12}$, Jianhua Zhang ${ }^{12}$, Juok Cho ${ }^{2}$, Daniel DiCara ${ }^{2}$, David Heiman ${ }^{2}$, Pei Lin ${ }^{2}$, William Mallard ${ }^{2}$, Douglas Voet ${ }^{2}$, Hailei Zhang ${ }^{2}$, Lihua Zou ${ }^{2}$, Michael S. Noble ${ }^{2}$, Michael S. Lawrence ${ }^{2}$, Gordon Saksena², Nils Gehlenborg ${ }^{2}$, Helga Thorvaldsdottir ${ }^{2}$ Jill Mesirov², Marc-Danie Nazaire ${ }^{2}$, Jim Robinson ${ }^{2}$, Gad Getz ${ }^{2,9,10}$; Memorial Sloan-Kettering Cancer Center William Lee ${ }^{4}$, B. Arman Aksoy ${ }^{4}$, Giovanni Ciriello ${ }^{4}$, Barry S. Taylor ${ }^{1}$, Gideon Dresdner ${ }^{4}$, Jianjiong Gao ${ }^{4}$, Benjamin Gross ${ }^{4}$, Venkatraman E. Seshan $^{4}$, Marc Ladanyi ${ }^{4}$, Boris Reva ${ }^{4}$, Rileen Sinha ${ }^{4}$, S. Onur Sumer ${ }^{4}$, Nils Weinhold $^{4}$ Nikolaus Schultz ${ }^{4}$, Ronglai Shen ${ }^{4}$, Chris Sander ${ }^{4}$; University of California, Santa Cruz/ Buck Institute Sam $\mathrm{Ng}^{18}$, Singer $\mathrm{Ma}^{18}$, Jingchun $\mathrm{Zhu}^{18}$, Amie Radenbaugh ${ }^{18}$, Joshua M. Stuart ${ }^{18}$, Christopher C. Benz ${ }^{21}$, Christina Yau ${ }^{21}$ \& David Haussler ${ }^{18,22}$; Oregon Health \& Sciences University Paul T. Spellman ${ }^{23}$; University of North Carolina, Chapel Hill Matthew D. Wilkerson ${ }^{11}$, Joel S. Parker ${ }^{11}$, Katherine A. Hoadley ${ }^{11}$, Patrick K. Kimes $^{11}$, D. Neil Hayes ${ }^{11}$, Charles M. Perou ${ }^{11}$; The University of Texas MD Anderson Cancer Center Bradley M. Broom ${ }^{12}$, Jing Wang ${ }^{12}$, Yiling Lu ${ }^{12}$, Patrick Kwok Shing Ng ${ }^{12}$ Lixia Diao $^{12}$, Lauren Averett Byers ${ }^{12}$, Wenbin Liu ${ }^{12}$, John V. Heymach ${ }^{12}$, Christopher I. Amos ${ }^{12}$, John N. Weinstein ${ }^{12}$, Rehan Akbani ${ }^{12}$, Gordon B. Mills ${ }^{12}$

Biospecimen core resource: International Genomics Consortium Erin Curley ${ }^{24}$, Joseph Paulauskis ${ }^{24}$, Kevin Lau ${ }^{24}$, Scott Morris ${ }^{24}$, Troy Shelton ${ }^{24}$, David Mallery ${ }^{24}$, Johanna Gardner ${ }^{24}$, Robert Penny ${ }^{24}$

Tissue source sites: Analytical Biological Service, Inc. Charles Saller ${ }^{25}$, Katherine Tarvin $^{25}$; Brigham \& Women's Hospital William G. Richards ${ }^{14}$; University of Alabama at Birmingham Robert Cerfolio ${ }^{26}$, Ayesha Bryant ${ }^{26}$; Cleveland Clinic:

Daniel P. Raymond ${ }^{27}$, Nathan A. Pennell ${ }^{27}$, Carol Farver ${ }^{27}$; Christiana Care Christine Czerwinski ${ }^{28}$, Lori Huelsenbeck-Dill ${ }^{28}$, Mary lacocca ${ }^{28}$, Nicholas Petrelli ${ }^{28}$, Brenda Rabeno ${ }^{28}$, Jennifer Brown ${ }^{28}$, Thomas Bauer ${ }^{28}$; Cureline Oleg Dolzhanskiy ${ }^{29}$ Olga Potapova ${ }^{29}$, Daniil Rotin ${ }^{29}$, Olga Voronina ${ }^{29}$, Elena Nemirovich-Danchenko ${ }^{29}$, Konstantin V. Fedosenko ${ }^{29}$; Emory University Anthony Gal ${ }^{30}$, Madhusmita Behera ${ }^{30}$ Suresh S. Ramalingam ${ }^{30}$, Gabriel Sica ${ }^{30}$; Fox Chase Cancer Center Douglas Flieder ${ }^{31}$ Jeff Boyd ${ }^{31}$, JoEllen Weaver ${ }^{31}$; ILSbio Bernard Kohl ${ }^{32}$, Dang Huy Ouoc Thinh ${ }^{32}$; Indiana University George Sandusky ${ }^{33}$; Indivumed Hartmut Juhl ${ }^{34}$; John Flynn Hospital Edwina Duhig ${ }^{35,36}$; Johns Hopkins University Peter $\mathrm{Ilei}^{6}$, Edward Gabrielson ${ }^{6}$, James Shin ${ }^{6}$, Beverly Lee ${ }^{6}$, Kristen Rodgers ${ }^{6}$, Dante Trusty ${ }^{6}$, Malcolm V. Brock $^{6}$; Lahey Hospital \& Medical Center Christina Williamson ${ }^{37}$, Eric Burks ${ }^{37}$, Kimberly Rieger-Christ ${ }^{37}$, Antonia Holway ${ }^{37}$, Travis Sullivan ${ }^{37}$; Mayo Clinic Dennis A Wigle $^{16}$, Michael K. Asiedu ${ }^{16}$, Farhad Kosari ${ }^{16}$; Memorial Sloan-Kettering Cancer Center William D. Travis ${ }^{4}$, Natasha Rekhtman ${ }^{4}$, Maureen Zakowski ${ }^{4}$, Valerie W. Rusch ${ }^{4}$ NYU Langone Medical Center Paul Zippile ${ }^{38}$, James Suh ${ }^{38}$, Harvey Pass ${ }^{38}$, Chandra Goparaju ${ }^{38}$, Yvonne Owusu-Sarpong ${ }^{38}$; Ontario Tumour Bank John M. S. Bartlett ${ }^{39}$ Sugy Kodeeswaran ${ }^{39}$, Jeremy Parfitt ${ }^{39}$, Harmanjatinder Sekhon ${ }^{39}$, Monique Albert ${ }^{39}$; Penrose St. Francis Health Services John Eckman ${ }^{40}$, Jerome B. Myers ${ }^{40}$; Roswell Park Cancer Institute Richard Cheney ${ }^{41}$, Carl Morrison ${ }^{41}$, Carmelo Gaudioso ${ }^{41}$; Rush University Medical Center Jeffrey A. Borgia ${ }^{42}$, Philip Bonomi ${ }^{42}$, Mark Pool ${ }^{42}$, Michael J. Liptay $^{42}$; St. Petersburg Academic University Fedor Moiseenko ${ }^{43}$, Irina Zaytseva ${ }^{43}$; Thoraxklinik am Universitätsklinikum Heidelberg, Member of Biomaterial Bank Heidelberg (BMBH) \& Biobank Platform of the German Centre for Lung Research (DZL) Hendrik Dienemann ${ }^{44}$, Michael Meister ${ }^{44}$, Philipp A. Schnabel ${ }^{45}$, Thomas R. Muley ${ }^{44}$; University of Cologne Martin Peifer ${ }^{46}$; University of Miami Carmen Gomez-Fernandez ${ }^{47}$, Lynn Herbert ${ }^{47}$, Sophie Egea ${ }^{47}$; University of North Carolina Mei Huang ${ }^{11}$, Leigh B. Thorne ${ }^{11}$, Lori Boice ${ }^{11}$, Ashley Hill Salazar ${ }^{11}$, William K. Funkhouser ${ }^{11}$, W. Kimryn Rathmell ${ }^{11}$; University of Pittsburgh Rajiv Dhir ${ }^{48}$, Samuel A Yousem $^{48}$, Sanja Dacic ${ }^{48}$, Frank Schneider ${ }^{48}$, Jill M. Siegfried ${ }^{48}$; The University of Texas MD Anderson Cancer Center Richard Hajek ${ }^{12}$; Washington University School of Medicine Mark A. Watson ${ }^{8}$, Sandra McDonald ${ }^{8}$, Bryan Meyers ${ }^{8}$; Queensland Thoracic Research Center Belinda Clarke ${ }^{35}$, Ian A. Yang ${ }^{35}$, Kwun M. Fong ${ }^{35}$, Lindy Hunter ${ }^{35}$, Morgan Windsor ${ }^{35}$, Rayleen V. Bowman ${ }^{35}$; Center Hospitalier Universitaire Vaudois Solange Peters ${ }^{49}$, Igor Letovanec ${ }^{49}$; Ziauddin University Hospital Khurram Z. Khan ${ }^{50}$

Data Coordination Centre Mark A. Jensen ${ }^{51}$, Eric E. Snyder ${ }^{51}$, Deepak Srinivasan ${ }^{51}$ Ari B. Kahn ${ }^{51}$, Julien Baboud ${ }^{51}$, David A. Pot ${ }^{51}$

Project team: National Cancer Institute Kenna R. Mills Shaw ${ }^{52}$, Margi Sheth ${ }^{52}$, Tanja Davidsen ${ }^{52}$, John A. Demchok ${ }^{52}$, Liming Yang ${ }^{52}$, Zhining Wang ${ }^{52}$, Roy Tarnuzzer ${ }^{52}$, Jean Claude Zenklusen ${ }^{52}$; National Human Genome Research Institute Bradley A Ozenberger ${ }^{53}$, Heidi J. Sofia ${ }^{53}$

Expert pathology panel William D. Travis ${ }^{4}$, Richard Cheney ${ }^{41}$, Belinda Clarke ${ }^{35}$, Sanja Dacic ${ }^{48}$, Edwina Duhig ${ }^{36,35}$, William K. Funkhouser ${ }^{11}$, Peter Illei ${ }^{6}$, Carol Farver ${ }^{27}$ Natasha Rekhtman ${ }^{4}$, Gabriel Sica ${ }^{30}$, James Suh ${ }^{38}$ \& Ming-Sound Tsao ${ }^{13}$

${ }^{1}$ University of California San Francisco, San Francisco, California 94158, USA. ${ }^{2}$ The Eli and Edythe L. Broad Institute, Cambridge, Massachusetts 02142, USA. ${ }^{3}$ Dana Farber Cancer Institute, Boston, Massachusetts 02115, USA. ${ }^{4}$ Memorial Sloan-Kettering Cancer Center New York, New York 10065, USA. ${ }^{5}$ University of Michigan, Ann Arbor, Michigan 48109, USA. ${ }^{6}$ Johns Hopkins University, Baltimore, Maryland 21287, USA. ${ }^{7}$ Baylor College of 


\section{RESEARCH ARTICLE}

Medicine, Houston, Texas 77030, USA. ${ }^{8}$ Washington University, St. Louis, Missouri 63108 USA. ${ }^{9}$ Harvard Medical School, Boston, Massachusetts 02115, USA. ${ }^{10}$ Massachusetts General Hospital, Boston, Massachusetts 02114, USA. ${ }^{11}$ University of North Carolina at Chapel Hill, Chapel Hill, North Carolina 27599, USA. ${ }^{12}$ University of Texas MD Anderson Cancer Center, Houston, Texas 77054, USA. ${ }^{13}$ Princess Margaret Cancer Centre, Toronto Ontario M5G 2M9, Canada. ${ }^{14}$ Brigham and Women's Hospital Boston, Massachusetts 02115, USA. ${ }^{15}$ BC Cancer Agency, Vancouver, British Columbia V5Z 4S6, Canada. ${ }^{16}$ Mayo Clinic, Rochester, Minnesota 55905, USA. ${ }^{17}$ University of Southern California, Los Angeles, California 90033, USA. ${ }^{18}$ University of California Santa Cruz, Santa Cruz, California 95064, USA. ${ }^{19}$ Massachusetts Institute of Technology, Cambridge,

Massachusetts 02142, USA. ${ }^{20}$ University of Kentucky, Lexington, Kentucky 40515, USA

${ }^{21}$ Buck Institute for Age Research, Novato, California 94945, USA. ${ }^{22}$ Howard Hughes Medical Institute, University of California Santa Cruz, Santa Cruz, California 95064, USA. ${ }^{23}$ Oregon Health and Science University, Portland, Oregon 97239, USA. ${ }^{24}$ International Genomics Consortium, Phoenix, Arizona 85004, USA. ${ }^{25}$ Analytical Biological Services, Inc., Wilmington, Delaware 19801, USA. ${ }^{26}$ University of Alabama at Birmingham, Birmingham, Alabama 35294, USA. ${ }^{27}$ Cleveland Clinic, Cleveland, Ohio 44195, USA

${ }^{28}$ Christiana Care, Newark, Delaware 19713, USA. ${ }^{29}$ Cureline, Inc., South San Francisco, California 94080, USA. ${ }^{30}$ Emory University, Atlanta, Georgia 30322, USA. ${ }^{31}$ Fox Chase Cancer Center, Philadelphia, Philadelphia 19111, USA. ${ }^{32}$ ILSbio, Chestertown, Maryland
21620, USA. ${ }^{33}$ Indiana University School of Medicine, Indianapolis, Indiana 46202, USA ${ }^{34}$ Individumed, Silver Spring, Maryland 20910, USA. ${ }^{35}$ The Prince Charles Hospital and the University of Queensland Thoracic Research Center, Brisbane, 4032, Australia.

${ }^{36}$ Sullivan Nicolaides Pathology \& John Flynn Hospital, Tugun 4680, Australia. ${ }^{37}$ Lahey Hospital and Medical Center, Burlington, Massachusetts 01805, USA. ${ }^{38}$ NYU Langone Medical Center, New York, New York 10016, USA. ${ }^{39}$ Ontario Tumour Bank, Ontario Institute for Cancer Research, Toronto, Ontario M5G 0A3, Canada. ${ }^{40}$ Penrose St. Francis Health Services, Colorado Springs, Colorado 80907, USA. ${ }^{41}$ Roswell Park Cancer Center, Buffalo, New York 14263, USA. ${ }^{42}$ Rush University Medical Center, Chicago, Illinois 60612, USA. ${ }^{43}$ St. Petersburg Academic University, St Petersburg 199034, Russia.

${ }^{44}$ Thoraxklinik am Universitätsklinikum Heidelberg, 69126 Heidelberg, Germany. ${ }^{45}$ University Heidelberg, 69120 Heidelberg, Germany. ${ }^{46}$ University of Cologne, 50931 Cologne, Germany. ${ }^{47}$ University of Miami, Sylvester Comprehensive Cancer Center, Miami, Florida 33136, USA. ${ }^{48}$ University of Pittsburgh, Pittsburgh, Pennsylvania 15213, USA. ${ }^{49} \mathrm{C} e n t e r$ Hospitalier Universitaire Vaudois, Lausanne and European Thoracic Oncology Platform, CH-1011 Lausanne, Switzerland. ${ }^{50}$ Ziauddin University Hospital, Karachi, 75300, Pakistan. ${ }^{51}$ SRA International, Inc., Fairfax, Virginia 22033, USA.

${ }^{52}$ National Cancer Institute, National Institutes of Health, Bethesda, Maryland 20892, USA. ${ }^{53}$ National Human Genome Research Institute, National Institutes of Health, Bethesda, Maryland 20892, USA. 
CORRIGENDUM

doi:10.1038/nature13879

Corrigendum: Comprehensive

molecular profiling of lung

adenocarcinoma

The Cancer Genome Atlas Research Network

Nature 511, 543-550 (2014); doi:10.1038/nature13385

In this Article, the surname of author Kristen Rodgers was incorrectly spelled Rogers. This error has been corrected in the HTML and PDF of the original paper. 
CORRECTION

https://doi.org/10.1038/s41586-018-0228-6

\section{Author Correction: Comprehensive molecular profiling of lung adenocarcinoma}

The Cancer Genome Atlas Research Network

Correction to: Nature https://doi.org/10.1038/nature13385, published online 9 July 2014; corrected online 8 October 2014.

In this Article, the Supplementary Table 7 iCLUSTER output column included incorrect cluster labels for the integrated subtypes presented in Fig. $5 c$. These changes affect only the iCLUSTER output column and do not affect the analysis or the conclusions of the work. The authors apologise for the error. Supplementary Table 7 has been corrected online, and the original incorrect table is provided as Supplementary Information to this Amendment for transparency.

Supplementary Information is available in the online version of this Amendment. 